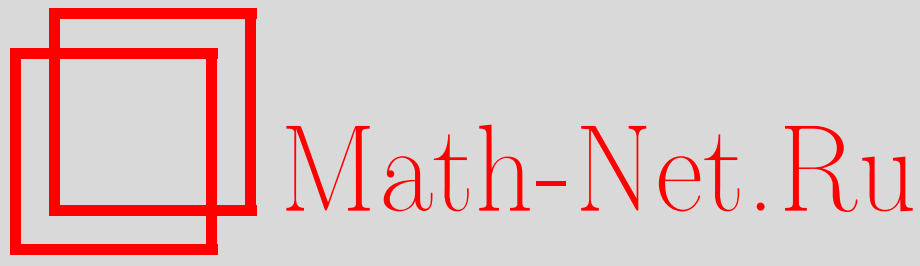

Обшероссийский математический портал

С. В. Конягин, О расстояниях между точками на плоскости, Матем. заметки, 2001, том 69, выпуск 4, 630-633

DOI: https://doi.org/10.4213/mzm691

Использование Общероссийского математического портала Math-Net.Ru подразумевает, что вы прочитали и согласны с пользовательским соглашением http://www . mathnet.ru/rus/agreement

Параметры загрузки:

IP : 3.95 .254 .165

26 апреля 2023 г., $16: 27: 46$ 


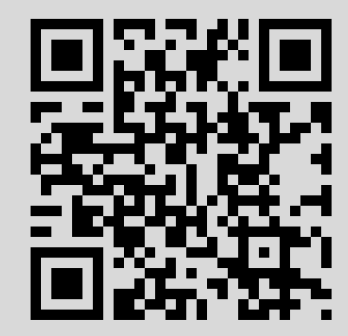




\section{О РАССТОЯНИЯХ МЕЖДДУ ТОЧКАМИ НА ПЛОСКОСТИ}

\section{С. В. Конягин}

1. Введение. Через $\{x\}$ мы будем обозначать дробную часть действительного числа $x$, a через $\|x\|$ - расстояние от $x$ до ближайшего целого числа. Расстояние между точками $P$ и $Q$ на плоскости обозначается $d(P, Q)$. Для $X>0$ и $\delta \in(0,1 / 2)$ через $N(X, \delta)$ обозначим максимальное количество точек $P_{1}, \ldots, P_{n}$, которые можно выбрать в круге радиуса $X$ так, чтобы выполнялось условие

$$
\left\|d\left(P_{i}, P_{j}\right)\right\| \geqslant \delta \quad(1 \leqslant i<j \leqslant n) .
$$

Легко показать, что равномерно по $X \geqslant 1$ и $\delta$ справедлива оценка $N(X, \delta)=O\left(X / \delta^{2}\right)$. П. Эрдеш предложил усилить это неравенство для любого $\delta$, т.е. показать, что $N(X, \delta)=o(X)$ при $X \rightarrow \infty$. Эта гипотеза была доказана А. Шаркози [1], установившшм, что $N(X, \delta)=O(X / \log \log X)$ для фиксированного $\delta$ и $X \geqslant 3$. Другая гипотеза Эрдеша, состоявшая в том, что $N(X, \delta) \rightarrow \infty$ при $X \rightarrow \infty$, доказана Р. Л. Грэмом. Наилучшие известные оценки снизу принадлежат Шаркози [2], доказавшему, что $N(X, \delta)>X^{c(\delta)}(c(\delta)>0)$; более того, $N(X, \delta)>X^{1 / 2-\delta^{1 / 7}}$ при $0<\delta \leqslant 1 /\left(6 \cdot 8^{4}\right)$ и $X \geqslant X(\delta)$. Последний резултат инициировал следующий вопрос Эрдеша и Грэма [3]: верно ли, что $N(X, \delta)<X^{1 / 2+\varepsilon}$ для любых $\varepsilon>0$ и $X \geqslant X(\delta, \varepsilon)$ ? Целью данной работы является положительный ответ на этот вопрос.

ТЕоремА. Для любого $\delta>0$ сущ,ествует число $C(\delta)$ maкое, что $N(X, \delta)<C(\delta) X^{1 / 2}$ при $X \geqslant 1$.

2. Начало доказательства теоремы. Пусть точки $P_{j}$ с координатами $\left(x_{j}, y_{j}\right)(j=1, \ldots$ $\ldots, n)$ расположены в круге радиуса $X$ и удовлетворяют условию (1). Для натурального числа $k$ и $\varphi \in[0,2 \pi)$ положим

$$
A_{k}(\varphi)=\sum_{j=1}^{n} e\left(k z_{j}(\varphi)\right)
$$

Работа выполнена при поддержке Российского фонда фундаментальных исследований, гранты № 99-01-00357 и № 00-15-96109. 
где $e(u)=\exp (2 \pi i u), z_{j}(\varphi)=x_{j} \cos \varphi+y_{j} \sin \varphi$. Пусть $d_{1}, \ldots, d_{m}-$ произвольные неотрицательные числа. Доказательство основано на неравенстве

$$
\sum_{k=1}^{m} d_{k} \int_{0}^{2 \pi}\left|A_{k}(\varphi)\right|^{2} d \varphi \geqslant 0
$$

Мы имеем

$$
\int_{0}^{2 \pi}\left|A_{k}(\varphi)\right|^{2} d \varphi=\sum_{i=1}^{n} \sum_{j=1}^{n} \int_{0}^{2 \pi} e\left(k\left(z_{i}(\varphi)-z_{j}(\varphi)\right)\right) .
$$

Пусть $x_{i}-x_{j}=d\left(P_{i}, P_{j}\right) \cos \varphi_{0}, y_{i}-y_{j}=d\left(P_{i}, P_{j}\right) \sin \varphi_{0}$. Тогда

$$
\begin{aligned}
\int_{0}^{2 \pi} e\left(k\left(z_{i}(\varphi)-z_{j}(\varphi)\right)\right) d \varphi & =\int_{0}^{2 \pi} e\left(k\left(d\left(P_{i}, P_{j}\right) \cos \left(\varphi-\varphi_{0}\right)\right)\right) d \varphi \\
& =2 \int_{0}^{\pi} \cos \left(2 \pi k d\left(P_{i}, P_{j}\right) \cos \varphi\right) d \varphi=2 \pi J_{0}\left(2 \pi k d\left(P_{i}, P_{j}\right)\right)
\end{aligned}
$$

(см. $[4$, с. 36$])$. Объединение (2), (3) и (4) дает

$$
\sum_{i=1}^{n} \sum_{j=1}^{n} \sum_{k=1}^{m} d_{k} 2 \pi J_{0}\left(2 \pi k d\left(P_{i}, P_{j}\right)\right) \geqslant 0
$$

Заметим, что

$$
\sum_{i=1}^{n} \sum_{k=1}^{m} d_{k} 2 \pi J_{0}\left(2 \pi k d\left(P_{i}, P_{i}\right)\right)=2 \pi n \sum_{k=1}^{m} d_{k}
$$

Нам нужно оценить вклад в (5) слагаемых с $i \neq j$. Из асимптотической формулы для $J_{0}$ :

$$
J_{0}(u)=\left(\frac{2}{\pi u}\right)^{1 / 2} \cos \left(u-\frac{\pi}{4}\right)+O\left(u^{-3 / 2}\right)
$$

(см. $[4$, с. 175]) вытекает, что

$$
2 \pi J_{0}(2 \pi v)=\left(\frac{2}{v}\right)^{1 / 2}(\cos (2 \pi v)+\sin (2 \pi v))+O\left(v^{-3 / 2}\right) .
$$

Подставляя последнюю оценку и (6) в (5), получаем

$$
\begin{aligned}
0 \leqslant & 2 \pi n \sum_{k=1}^{m} d_{k}+\sum_{i=1}^{n} \sum_{j \neq i} d\left(P_{i}, P_{j}\right)^{-1 / 2} \sum_{k=1}^{m}\left(\frac{2}{k}\right)^{1 / 2} d_{k}\left(\cos \left(2 \pi k d\left(P_{i}, P_{j}\right)\right)+\sin \left(2 \pi k d\left(P_{i}, P_{j}\right)\right)\right) \\
& +O\left(\sum_{i=1}^{n} \sum_{j \neq i} d\left(P_{i}, P_{j}\right)^{-3 / 2}\right)
\end{aligned}
$$

где постоянная в $O$ зависит от коэффициентов $d_{1}, \ldots, d_{m}$. Коэффициенты $d_{k}$, при которых второе слагаемое в правой части (7) мало, мы будем подбирать с помощью нижеследующей леммы. 


\section{3. Тригонометрические полиномы со специальными свойствами.}

ЛЕмма 1. Для любого $\delta \in(0,1 / 2)$ существует тригонометрический полином

$$
T(x)=\sum_{k=1}^{m} c_{k} \cos (k x)
$$

c неотрицательными коэффициентами $c_{k}$ такой, что сопряженный полином

$$
\widetilde{T}(x)=\sum_{k=1}^{m} c_{k} \sin (k x)
$$

удовлетворяет неравенству

$$
-A=\max _{x \in[2 \pi \delta, 2 \pi(1-\delta)]}(T(x)+|\tilde{T}(x)|)<0 .
$$

ДокАЗАТЕЛЬСтво. Определим функцию комплексного переменного $z$ :

$$
f(z)=\frac{z}{(1-z)^{2}}
$$

Для любого $z \neq 1,|z|=1$, имеем

$$
f(z)=\frac{1}{z+1 / z-2}=\frac{1}{z+\bar{z}-2}=\frac{1}{2 \operatorname{Re} z-2}<0 .
$$

Кроме того, функции $f\left(u e^{i x}\right)$ равномерно сходятся к $f\left(e^{i x}\right)$ на $[2 \pi \delta, 2 \pi(1-\delta)]$ при $u \rightarrow 1$. Следовательно, для действительного $u<1$, достаточно близкого к 1 , имеет место неравенство

$$
\max _{x \in[2 \pi \delta, 2 \pi(1-\delta)]}\left(\operatorname{Re} f\left(u e^{i x}\right)+\left|\operatorname{Im} f\left(u e^{i x}\right)\right|\right)<0 .
$$

Рассмотрим разложение Тейлора

$$
\sum_{k=1}^{\infty} c_{k} z^{k}
$$

функции $f(u z)$, и пусть

$$
S_{m}(z)=\sum_{k=1}^{m} c_{k} z^{k}
$$

Из (8) и равномерной сходимости частных сумм $S_{m}(z)$ к $f(u z)$ в единичном круге мы имеем

$$
\max _{x \in[2 \pi \delta, 2 \pi(1-\delta)]}\left(\operatorname{Re} S_{m}\left(u e^{i x}\right)+\left|\operatorname{Im} S_{m}\left(u e^{i x}\right)\right|\right)<0
$$

Таким образом, тригонометрический полином $T(x)=\operatorname{Re} S_{m}\left(u e^{i x}\right)$ удовлетворяет требуемому неравенству. Остается заметить, что все коэффициенты $c_{k}$ положительны, и лемма доказана. 
4. Завершение доказательства теоремы. Возьмем $d_{k}=(k / 2)^{1 / 2} c_{k}(k=1, \ldots, m)$, где $m$ и $c_{k}$ определены в лемме 1 . С учетом (1) мы имеем

$$
\begin{aligned}
& \sum_{i=1}^{n} \sum_{j \neq i} d\left(P_{i}, P_{j}\right)^{-1 / 2} \sum_{k=1}^{m}\left(\frac{2}{k}\right)^{1 / 2} d_{k}\left(\cos \left(2 \pi k d\left(P_{i}, P_{j}\right)\right)+\sin \left(2 \pi k d\left(P_{i}, P_{j}\right)\right)\right) \\
& \quad \leqslant \sum_{i=1}^{n} \sum_{j=1}^{n}(2 X)^{-1 / 2}(-A)=-A(2 X)^{-1 / 2} n^{2}
\end{aligned}
$$

Для любого $i=1, \ldots, n$ и $u>0$ обозначим $N_{i}(u)=\left|\left\{j \neq i: d\left(P_{i}, P_{j}\right) \leqslant u\right\}\right|$. Заметим, что $N_{i}(u) \leqslant N(u, \delta)$. Мы используем оценку $N(u, \delta)=O(1+u)$, более слабую, чем в [1]; здесь и ниже постоянные в $O$ зависят от $\delta$. Мы имеем

$$
\begin{aligned}
\sum_{j \neq i} d\left(P_{i}, P_{j}\right)^{-3 / 2} & =\int_{\delta}^{\infty} u^{-3 / 2} d N_{i}(u)=-\int_{\delta}^{\infty} N_{i}(u) d\left(u^{-3 / 2}\right) \\
& =\int_{\delta}^{\infty} O(u) \frac{3}{2} u^{-5 / 2} d u=O(1)
\end{aligned}
$$

Из (7), (9) и последней оценки следует, что

$$
O(n)-A(2 X)^{-1 / 2} n^{2} \geqslant 0
$$

т.е. $n=O\left(X^{1 / 2}\right)$, что и требовалось доказать.

Автор благодарен профессорам А. Шаркози и В. Тотику за интерес к работе и полезные обсуждения.

\section{СПИСОК ЦИТИРОВАННОЙ ЛИТЕРАТУРЫ}

1. Sárközy A. // Studia Sci. Math. Hungar. 1976. V. 11. P. 37-50. 2. Sárközy A. // Studia Sci. Math. Hungar. 1976. V. 11. P. 105-111. 3. Erdös P., Graham R. L. Old and New Problems and Results in Combinatorial Number Theory. Geneva, 1980. 4. Коренев В. Г. Введение в теорию бесселевых функций. М.: Наука, 1971.

Московский государственный университет им. М.В. Ломоносова 